\title{
ON DIRICHLET SERIES ATTACHED TO CUSP FORMS AND THE SIEGEL-ZERO
}

\author{
by F. GRUPP
}

(Received 26 November, 1982)

1. Introduction and statement of results. Let $k$ be an even integer greater than or equal to 12 and $f$ an nonzero cusp form of weight $k$ on $\operatorname{SL}(2, \mathbb{Z})$. We assume, further, that $f$ is an eigenfunction for all Hecke-Operators and has the Fourier expansion

$$
f(z)=\sum_{n=1}^{\infty} a(n) e^{2 \pi i n z}, \text { where } a(1)=1 .
$$

For every Dirichlet character $\chi \bmod q$ we define

$$
L_{f}(s, \chi)=\sum_{n=1}^{\infty} \chi(n) a(n) n^{-s} .
$$

Then the series in (1.1) is absolutely convergent for $\operatorname{Re} s>(k+1) / 2 . L_{f}(s, \chi)$ can be analytically continued as an entire function. For a primitive character $\chi, L_{f}(s, \chi)$ satisfies the following functional equation (cf. [2])

$$
\left(\frac{2 \pi}{q}\right)^{-s} \Gamma(s) L_{f}(s, \chi)=\left(\frac{2 \pi}{q}\right)^{-(k-s)} \Gamma(k-s) \varepsilon(\chi) L_{f}(k-s, \bar{\chi})
$$

where $|\varepsilon(\chi)|=1$. For a nonprimitive character $\chi$ the analytical continuation for $L_{f}(s, \chi)$ will be given later (Lemma 2).

We note, that throughout the paper $A, a$ denote positive constants but not always the same, whereas $a_{1}, a_{2}, \ldots, b_{1}, b_{2}, \ldots, c_{1}, c_{2}, \ldots$ are the same positive constants. $\zeta(s)$ is Riemann's zeta-function and $p$ always denotes a prime.

Rankin [7] proved that $L_{f}\left(s, \tilde{X}_{0}\right) \neq 0$ for $s=(k+1) / 2+i t$, except possibly for $t=0$, where $\tilde{\chi}_{0}$ is the principal character mod 1. Ogg [4] later proved that $L_{f}\left((k+1) / 2, \tilde{\chi}_{0}\right) \neq 0$, if the Petersson conjecture holds. This conjecture has meanwhile been proved by Deligne [1]. We shall first generalize this result in the following way:

THEOREM 1. Let $z \geqq 2$ and $L_{f}(s, \chi)$ as above. Then there is a $c_{1}$ independent of $z$, such that, for

$$
\sigma \geqq 1-\frac{c_{1}}{\log (z(|t|+2))}, \quad L_{f}\left(s+\frac{k-1}{2}, \chi\right) \neq 0
$$

for all $\chi \bmod q$ with $q \leqq z$, with the possible exception of those $L_{f}(s+(k-1) / 2, \chi)$ with character $\chi$ equivalent to a unique primitive real character $\chi^{*} \bmod q^{*}\left(\chi^{*}=\chi^{*}(z), q^{*}=\right.$ $\left.q^{*}(z)\right)$. These exceptional $L_{f}(s+(k-1) / 2, \chi)$, if they exist, all have the same zero $\hat{\sigma}$. This zero $\hat{\sigma}$ is real, simple and different from 1 .

Glasgow Math. J. 25 (1984) 107-119. 
Theorem 1 also holds for Dirichlet $L$-series (cf. [5], IV). The exceptional zero for the Dirichlet $L$-series is usually called the Siegel zero. Let us call $\hat{\sigma}$ the Siegel zero for the cusp form $f$. It is not known whether the Siegel zero exists or not, nor whether the Siegel zero for the cusp form $f$ exists or not. The following theorem shows that both can not exist.

THEOREM 2. Let $z \geqq 2, L_{f}(s, \chi)$ be as before and $L(s, \chi)$ a Dirichlet $L$-series. Then there is a $c_{2}$ independent of $z$, such that, for

$$
\sigma \geqq 1-\frac{c_{2}}{\log (z(|t|+2))}, \quad L(s, \chi) \neq 0 \quad \text { and } \quad L_{f}\left(s+\frac{k-1}{2}, \chi\right) \neq 0
$$

for all $\chi \bmod q$ with $q \leqq z$ with the following possible exception: for primitive characters $\chi$ at most one of the excepted functions $L_{f}(s+(k-1) / 2, \chi)$ of Theorem 1 and the excepted functions $L(s, \chi)$ of [5], IV, Satz 6.9 can have a zero in

$$
\sigma \geqq 1-\frac{c_{2}}{\log (z(|t|+2))} .
$$

As a special case of Theorem 2 we have the following result:

There is a $c_{2}$ independent of $z$ such that either

$$
L_{f}\left(s+\frac{k-1}{2}, \chi\right) \neq 0 \quad \text { for } \quad \sigma \geqq 1-\frac{c_{2}}{\log (z(|t|+2))} \text { for all } \chi \bmod q \text { with } q \leqq z,
$$

or

$$
L(s, \chi) \neq 0 \quad \text { for } \quad \sigma \geqq 1 \frac{c_{2}}{\log (z(|t|+2))} \quad \text { for all } \chi \bmod q \text { with } q \leqq z .
$$

The zero-free domains of $L(s, \chi)$ and $L_{f}(s, \chi)$ allor $\iota s$ to prove the following results:

THEOREM 3. Let $x \geqq 2,(l, q)=1$ and $B$ some constant. Then there is a constant $c_{3}$ such that either

$$
\text { (i) } \sum_{\substack{p \leqq x \\ p=1 \bmod q}} \log p-\frac{x}{\varphi(q)}=O\left(x \exp \left\{-c_{3} \sqrt{ } \log x\right\}\right)
$$

uniformly for $1 \leqq q \leqq \exp \{B \sqrt{ } \log x\}$, or

$$
\text { (ii) } \sum_{\substack{p \leq x \\ p=1 \bmod q}} a(p) p^{-(k-1) / 2} \log p=O\left(x \exp \left\{-c_{3} \sqrt{ } \log x\right\}\right)
$$

uniformly for $1 \leqq q \leqq \exp \{B \sqrt{ } \log x\}$, or both (i) and (ii) hold true.

REMARKS.

(1) The particular alternative occurring in Theorem 3 may depend on the choice of $x$, because the exceptional character in Theorem 2 depends on $z$.

(2) Note that in both (i) and (ii) the conditions $q \leqq \exp \{B \log x\}$ may be dropped since otherwise the results become trivial. 
THEOREM 4. There is a constant $c_{4}$ such that

$$
\sum_{\substack{p \leqq x \\ p=l \bmod q}} a(p) p^{-(k-1) / 2} \log p=O\left(x \exp \left\{-c_{4} \sqrt{ } \log x\right\}\right) .
$$

A proof for Theorem 4 in the case $q=1$ was given by Moreno [3]. In $\$ 2$ we prove several Lemmas from which we deduce the truth of Theorems 1 and 2 . Theorems 3 and 4 are proved in $\$ 3$. The paper contains part of the author's dissertation. He takes this opportunity to thank Professor Dr. H.-E. Richert for his advice and encouragement.

\section{Proofs of Theorem 1 and Theorem 2.}

Lemma 1. Let $\chi$ be a Dirichlet character $\bmod q$. Then

$$
L_{f}(s, \chi) \neq 0 \text { for } \sigma>\frac{k+1}{2} .
$$

Proof. We have $a(p) \in \mathbb{R}$ and $|a(p)| \leqq 2 p^{(k-1) / 2}$ for all primes $p$ (cf. [1]). Hence $\theta_{p}(a)$ is uniquely defined by

$$
\cos \theta_{\mathrm{p}}(a)=\frac{1}{2} a(p) p^{-(k-1) / 2} \quad\left(0 \leqq \theta_{\mathrm{p}}(a) \leqq \pi\right) .
$$

For $L_{f}(s, \chi)$ the following Euler product representation holds:

$$
L_{f}(s, \chi)=\prod_{p}\left\{\left(1-\frac{\chi(p) e^{i \theta_{p}(a)}}{p^{s-(k-1) / 2}}\right)\left(1-\frac{\chi(p) e^{-i \theta_{p}(a)}}{p^{s-(k-1) / 2}}\right)\right\}^{-1} \quad\left(\sigma>\frac{k+1}{2}\right) .
$$

Rankin proved this formula for $\chi=\tilde{\chi}_{0} \bmod 1$ in [7]. For arbitrary character $\chi \bmod q$ the proof proceeds similarly, using the fact that $\chi(n) \chi(m)=\chi(n m)$ for all $n, m \in \mathbb{N}$. That $L_{f}(s, \chi) \neq 0$ for $\sigma>(k+1) / 2$ now follows from $(2.1)$.

LEMMA 2. Let $\chi$ be $\bmod q, q^{*}$ the conductor of $\chi$ and $\chi^{*} \bmod q^{*}$ the primitive character inducing $\chi$. Then

$$
L_{f}(s, \chi)=L_{f}\left(s, \chi^{*}\right) \prod_{p \mid q}\left\{\left(1-\frac{\chi^{*}(p) e^{i \theta_{p}(a)}}{p^{s-(k-1) / 2}}\right)\left(1-\frac{\chi^{*}(p) e^{-i \theta_{p}(a)}}{p^{s-(k-1) / 2}}\right)\right\} ;
$$

$L_{f}(s, \chi)$ and $L_{f}\left(s, \chi^{*}\right)$ have the same zeros for $\sigma>(k-1) / 2$.

Proof. For $\sigma>(k+1) / 2$ (2.2) follows from (2.1) and for $\sigma>(k-1) / 2$ by analytical continuation. Since the finite product in (2.2) is different from zero for $\sigma>(k-1) / 2$, $L_{f}(s, \chi)$ and $L_{f}\left(s, \chi^{*}\right)$ have the same zeros for $\sigma>(k-1) / 2$.

In order to prove zero-free regions for $L_{f}(s, \chi)$ it is now sufficient from now on to take $\chi$ primitive $\bmod q$.

Lemma 3. Let $0<\eta<\frac{1}{2},(k-1) / 2-\eta \leqq \sigma \leqq(k+1) / 2+\eta, t_{1}=|t|+2$. Then

$$
\begin{gathered}
\left|L_{f}(s, \chi)\right| \leqq A \zeta(1+\eta)(q|1+s|)^{(k+1) / 2-\sigma+\eta} \\
\left|L_{f}(s, \chi)\right| \leqq A \log \left(q t_{1}\right) \quad \text { for } \quad \frac{k+1}{2}-\frac{a}{\log \left(q t_{1}\right)} \leqq \sigma \leqq \frac{k+3}{2}, \quad a \leqq 4 .
\end{gathered}
$$


Proof. We take $Q=(k-1) / 2$ and $s=w-(k-1) / 2$ in Lemma 3 of [6]. This gives

$$
\left|\frac{\Gamma(k-w)}{\Gamma(w)}\right| \leqq|w+1|^{k-2 u} \text { for } \frac{k}{2}-1 \leqq \operatorname{Re} w=u \leqq \frac{k}{2}
$$

For $\eta>0$

$$
\left|L_{f}\left(\frac{k+1}{2}+\eta+i t, \chi\right)\right| \leqq\left\{\sum_{n=1}^{\infty} \frac{a^{2}(n)}{n^{k+\eta}}\right\}^{1 / 2} \zeta^{1 / 2}(1+\eta) \leqq A \zeta(1+\eta)
$$

In the last step we used a result due to Rankin [8]. Then, by (1.2), (2.5) and (2.6),

$$
\left|L_{f}\left(\frac{k-1}{2}-\eta+i t, \chi\right)\right| \leqq A q^{-1-2 \eta}\left|\frac{k+1}{2}-\eta+i t\right|^{1+2 \eta} \zeta(1+\eta) .
$$

Choosing now $a=(k-1) / 2-\eta, b=(k+1) / 2+\eta, Q=1$ in Theorem 2 of [6] we obtain (2.3). We now take

$$
\eta=\frac{1}{\log \left(q t_{1}+7\right)}\left(\leqq \frac{1}{2}\right), \quad \sigma \geqq \frac{k+1}{2}-\frac{a}{\log \left(q t_{1}\right)}
$$

in (2.3). Then (2.4) follows for $\sigma \leqq(k+1) / 2+1 / \log \left(q t_{1}+7\right)$. For $(k+1) / 2 \geqq \sigma>$ $(k+1) / 2-1 / \log \left(q t_{1}+7\right)(2.4)$ follows by $(2.6)$ since $\zeta(1+\eta) \leqq(2 / \eta)$ for $0<\eta \leqq \frac{1}{2}$.

LEMmA 4. There is an $a \leqq 1$, such that

$$
L_{f}(s, \chi) \neq 0 \quad \text { for } \quad \sigma \geqq \frac{k+1}{2}-\frac{a}{\log (q|t|)}, \quad|t| \geqq 3 \text {, and all } \chi \bmod q \text {. }
$$

Proof. Taking the logarithms and differentiating (2.1), we obtain

Defining

$$
\frac{L_{f}^{\prime}}{L_{f}}(s, \chi)=-\sum_{p, m \geqq 1} \frac{2 \chi\left(p^{m}\right) \cos \left(m \theta_{p}(a)\right)}{p^{m(s-(k-1) / 2)}} \log p \text { for } \sigma>\frac{k+1}{2} .
$$

$$
\psi_{f}(s, \chi)=\prod_{p}\left(\left(1-\frac{\chi(p) e^{2 i \theta_{p}(a)}}{p^{s-k+1}}\right)\left(1-\frac{\chi(p) e^{-2 i \theta_{p}(a)}}{p^{s-k+1}}\right)\right)^{-1} \quad(\sigma>k),
$$

we obtain

$$
\frac{\psi_{f}^{\prime}}{\psi_{f}}\left(s+\frac{k-1}{2}, \chi\right)=-\sum_{p, m \geqq 1} \frac{2 \chi\left(p^{\prime \prime \prime}\right) \cos \left(2 m \theta_{p}(a)\right)}{p^{m(s-(k-1) / 2)}} \log p \quad\left(\sigma>\frac{k+1}{2}\right) .
$$

For Dirichlet $L$-series we have

$$
\frac{L^{\prime}}{L}\left(s-\frac{k-1}{2}, \chi\right)=-\sum_{p, m \geqq 1} \frac{\chi\left(p^{m}\right)}{p^{m(s-(k-1) / 2)}} \log p \quad\left(\sigma>\frac{k+1}{2}\right) .
$$


If $\chi_{0}$ is the principal character $\bmod q$ then $(2.8),(2.9)$ and $(2.10)$ give

$$
\begin{aligned}
& \operatorname{Re}\left\{-2 \frac{L^{\prime}}{L}\left(\sigma-\frac{k-1}{2}, \chi_{0}\right)-\frac{1}{2} \frac{\psi_{f}^{\prime}}{\psi_{f}}\left(\sigma+\frac{k-1}{2}, \chi_{0}\right)\right. \\
- & \left.\frac{L^{\prime}}{L}\left(\sigma-\frac{k-1}{2}+2 i t, \chi^{2}\right)-2 \frac{L_{f}^{\prime}}{L_{f}}(\sigma+i t, \chi)\right\} \\
= & \sum_{p, m \geqslant 1} \frac{\log p}{p^{m(\sigma-(k-1) / 2)}}\left\{2 \chi_{0}\left(p^{m}\right)+\chi_{0}\left(p^{m}\right) \cos \left(2 m \theta_{p}(a)\right)+\operatorname{Re}\left(e^{-2 i m t \log p} \chi^{2}\left(p^{m}\right)\right)\right. \\
& \left.+4 \operatorname{Re}\left(e^{-i m \log p} \chi\left(p^{m}\right)\right) \cos \left(m \theta_{p}(a)\right)\right\} \geqq 0 \quad(\sigma>(h+1) / 2),
\end{aligned}
$$

for the expression in brackets on the right of (2.11) is not negative. Let now $\rho=\beta+i \gamma$ be a zero of $L_{f}(s, \chi)$ and put

$$
\sigma_{0}=\frac{k+1}{2}+\frac{a_{1}}{\log (q \gamma)},
$$

where $a_{1} \leqq 1, \gamma \geqq 3$ and $s_{0}=\sigma_{0}+i \gamma$. Then we have (cf. [5])

and

$$
-\frac{L^{\prime}}{L}\left(\sigma_{0}-\frac{k-1}{2}, \chi_{0}\right) \leqq-\frac{\zeta^{\prime}}{\zeta}\left(\sigma_{0}-\frac{k-1}{2}\right) \leqq \frac{5}{4 a_{1}} \log (q \gamma)
$$

$$
-\operatorname{Re} \frac{L^{\prime}}{L}\left(\sigma_{0}-\frac{k-1}{2}+2 i \gamma, \chi^{2}\right)<A\left\{\log (q \gamma)+\log \frac{1}{a_{1}}\right\}
$$

Let further $\mu_{a}(n)$ be a multiplicative function defined by

for all primes $p$. Then we have

$$
\mu_{a}(n)= \begin{cases}-a(p) & (n=p), \\ p^{k-1} & \left(n=p^{2}\right), \\ 0 & \left(n=p^{\nu}, \nu \geqq 3\right) .\end{cases}
$$

and

$$
\sum_{t \mid n} \mu_{a}(t) a\left(\frac{n}{t}\right)= \begin{cases}1 & (n=1) \\ 0 & (n>1)\end{cases}
$$

Hence

$$
\left|\mu_{a}(n)\right| \leqq d(n) n^{(k-1) / 2}, \quad \text { where } \quad d(n)=\sum_{i \mid n} 1
$$

$$
\frac{1}{\left|L_{f}\left(s_{0}, \chi\right)\right|} \leqq \sum_{n=1}^{\infty} \frac{\left|\mu_{a}(n)\right|}{n^{\sigma_{o}}} \leqq \zeta^{2}\left(1+\frac{a_{1}}{\log (q \gamma)}\right) \leqq \frac{4}{a_{1}^{2}} \log ^{2}(q \gamma) .
$$

This together with the results of Lemma 3 then gives

$$
\left|\frac{L_{f}(s, \chi)}{L_{f}\left(s_{0}, \chi\right)}\right| \leqq \frac{1}{a_{1}^{2}}(q \gamma)^{\mathrm{A}} \text { for } \quad\left|s-s_{0}\right| \leqq \frac{1}{2}
$$


If now $\rho=\beta+i \gamma$ is a zero for $L_{f}(s, \chi)$ with $\left|\rho-s_{0}\right|=\sigma_{0}-\beta \leqq \frac{1}{4}$ we have ([5] Anhang)

$$
-\operatorname{Re} \frac{L_{f}^{\prime}}{L_{f}}\left(\sigma_{0}+i \gamma, \chi\right) \leqq A\left(\log (q \gamma)+\log \frac{1}{a_{1}}\right)-\frac{1}{\sigma_{0}-\beta} .
$$

If $\chi_{0}$ is the principal character $\bmod q$ we further obtain

where

$$
\psi_{f}\left(\sigma+\frac{k-1}{2}, \chi_{0}\right)=\psi_{f}\left(\sigma+\frac{k-1}{2}\right) \prod_{p \mid a}\left\{\left(1-\frac{e^{2 i \theta_{p}(a)}}{p^{\sigma-(k-1) / 2}}\right)\left(1-\frac{e^{-2 i \theta_{p}(a)}}{p^{\sigma-(k-1) / 2}}\right)\right\}
$$

$$
\psi_{f}\left(\sigma+\frac{k-1}{2}\right)=\prod_{\mathbf{p}}\left\{\left(1-\frac{e^{2 \mathbf{i}_{\mathrm{p}}(a)}}{p^{\sigma-(k-1) / 2}}\right)\left(1-\frac{e^{-2 i \boldsymbol{\theta}_{\mathrm{n}}(a)}}{p^{\sigma-(k-1) / 2}}\right)\right\}^{-1} .
$$

Taking logarithms and differentiating we deduce that

$$
\frac{\psi_{f}^{\prime}}{\psi_{f}}\left(\sigma+\frac{k-1}{2}, \chi_{0}\right)=\frac{\psi_{f}^{\prime}}{\psi_{f}}\left(\sigma+\frac{k-1}{2}\right)+\sum_{\substack{p \mid a \\ m \geqq 1}} \frac{2 \cos \left\{2 m \theta_{p}(a)\right\}}{p^{m(\sigma-(k-1) / 2)}} \log p .
$$

Using results of Rankin [9] concerning $\psi_{f}(s)$ and the inequality

$$
\left|\sum_{\substack{p \mid q \\ m \geqq 1}} \frac{2 \cos \left(2 m \theta_{p}(a)\right)}{p^{m(\sigma-(k-1) / 2)}} \log p\right| \leqq A \log \log (q+2) \quad \text { for } \quad \sigma \geqq \frac{k+1}{2}
$$

we obtain

$$
-\frac{1}{2} \frac{\psi_{f}^{\prime}}{\psi_{f}}\left(\sigma_{0}+\frac{k+1}{2}, \chi_{0}\right) \leqq \frac{\zeta^{\prime}}{\zeta}\left(\sigma_{0}-\frac{k-1}{2}\right)+\frac{5}{8\left(\sigma_{0}-\frac{k-1}{2}\right)},
$$

for $a_{1}$ sufficiently small. Take $\sigma=\sigma_{0}, t=\gamma$ in (2.11); then (2.12), (2.13), (2.14) and (2.15) give

Hence

$$
0 \leqq \frac{15}{8 a_{1}} \log (q \gamma)+A\left(\log (q \gamma)+\log \frac{1}{a_{1}}\right)-\frac{2}{\sigma_{0}-\beta} .
$$

$$
\beta \leqq \frac{k+1}{2}-\frac{a_{2}}{\log (q \gamma)} \text { for } \gamma \geqq 3
$$

and $a_{2}$ sufficiently small. The inequality

$$
\beta \leqq \frac{k+1}{2}-\frac{a_{3}}{\log (q|\gamma|)} \text { for } \gamma \leqq-3
$$

follows since $L_{f}(\bar{s}, \chi)=\overline{L_{f}(s, \bar{\chi})}$.

Lemma 5. Let $\chi^{2} \neq \chi_{0}$. Then we have

$$
L_{f}(s, \chi) \neq 0 \quad \text { for } \quad \sigma \geqq \frac{k+1}{2}-\frac{a}{\log q}, \quad|t| \leqq 3 .
$$


Proof. The proof runs as the proof of Lemma 4, taking now

instead of $\sigma_{0}$.

$$
\sigma_{1}=\frac{k+1}{2}+\frac{a_{3}}{\log q}
$$

We have for $\chi_{0} \bmod q$

from (2.2) and

$$
L_{f}\left(s, \chi_{0}\right) \neq 0 \quad \text { for } \quad \sigma \geqq \frac{k+1}{2}-\frac{a_{4}}{\log (|t|+3)}, \quad t \in \mathbb{R}
$$

$$
\zeta_{f}(s)=L_{f}\left(s, \bar{\chi}_{0}\right) \neq 0 \quad \text { for } \quad \sigma \geqq \frac{k+1}{2}-\frac{a_{4}}{\log (|t|+3)}, \quad t \in \mathbb{R}
$$

where $\tilde{\chi}_{0}(n)=1$ for all $n$ (cf. [3]).

Lemma 6. Let $\chi^{2}=\chi_{0}, \chi \neq \chi_{0}$. Then we have

$$
L_{f}(s, \chi) \neq 0 \quad \text { for } \quad \sigma \geqq \frac{k+1}{2}-\frac{a}{\log q}, \quad 0<|t| \leqq 3 .
$$

Proof. We follow the proof of Lemma 4, taking $\sigma_{2}=(k+1) / 2+a_{5} / \log q$ instead of $\sigma_{0}$. Then the estimates (2.12), (2.14) and (2.15) still hold with $\sigma_{2}$ instead of $\sigma_{0}, a_{5}$ instead of $a_{1}$ and $q$ instead of $q \gamma$. If $\rho=\beta+i \gamma$ is a zero of $L_{f}(s, \chi)$ with $a / \log q \leqq \gamma \leqq 3$, then we have

$$
-\operatorname{Re} \frac{L^{\prime}}{L}\left(\sigma_{2}-\frac{k-1}{2}+2 i \gamma, x_{0}\right) \leqq A \log q .
$$

As in the proof of Lemma 4 we finally obtain

$$
\beta \leqq \frac{k+1}{2}-\frac{a}{\log q} \text { where } \frac{a}{\log q} \leqq|\gamma| \leqq 3 .
$$

If $\rho=\beta+i \gamma$ is a zero of $L_{f}(s, \chi)$ with

$$
0<\gamma<\frac{a_{6}}{\log q} \text { and } \frac{k+1}{2} \geqq \beta \geqq \frac{k+1}{2}-\frac{a_{7}}{\log q},
$$

$\bar{\rho}=\beta-i \gamma$ is also a zero of $L_{f}(s, \chi)$, since $L_{f}(\bar{s}, \chi)=\overline{L_{f}(s, \chi)}$ and both zeros are in $\left|s-s_{3}\right| \leqq \frac{1}{4}$ for $s_{3}=(k+1) / 2+a_{8} / \log q+i \gamma$, if $a_{6}, a_{7}, a_{8}$ are sufficiently small. Then we obtain (cf. [5])

$$
-\operatorname{Re} \frac{L_{f}^{\prime}}{L_{f}}\left(\sigma_{3}+i \gamma, \chi\right)<A\left(\log q+\log \frac{1}{a_{8}}\right)-\frac{1}{\sigma_{3}-\beta}-\frac{\sigma_{3}-\beta}{\left(\sigma_{3}-\beta\right)^{2}+4 \gamma^{2}} .
$$

On the other hand we have

$$
\begin{aligned}
\operatorname{Re} \frac{L_{f}^{\prime}}{L_{f}}\left(\sigma_{3}+i \gamma, \chi\right) \leqq & \left\{\sum_{p, m \geqq 1} \frac{4 \cos ^{2}\left(m \theta_{p}(a)\right)}{p^{m\left(\sigma_{3}-(k-1) / 2\right)}} \log p\right\}^{1 / 2} \\
& \times\left\{\sum_{p, m \geqq 1} \frac{\log p}{p^{m\left(\sigma_{3}-(k-1) / 2\right)}}\right\}^{1 / 2} \leqq \frac{5}{4\left(\sigma_{3}-\frac{k+1}{2}\right)}=\frac{5}{4 a_{8}} \log q
\end{aligned}
$$


for $a_{8}$ sufficiently small (cf. [9]). This together with (2.20) gives

$$
\frac{k+1}{2}-\beta+\frac{a_{8}}{\log q}<\left(\frac{1}{2 a_{8}}+\frac{a_{7}}{a_{8}^{2}}\right)\left\{\left(a_{7}+a_{8}\right)^{2}+4 a_{6}^{2}\right\} \frac{1}{\log q} .
$$

If $a_{6}$ and $a_{7}$ are small enough, the right-side of $(2.21)$ is smaller than $a_{8} / \log q$. Hence $\beta>(k+1) / 2$, which contradicts Lemma 1 . Hence

$$
L_{f}(s, \chi) \text { has no zero for } \frac{k+1}{2} \geqq \beta \geqq \frac{k+1}{2}-\frac{a_{7}}{\log q}, \quad 0<|\gamma|<\frac{a_{6}}{\log q} .
$$

This together with (2.19) proves (2.18).

Lemma 7. Let $\chi^{2}=\chi_{0}, \chi \neq \chi_{0}$. Then $L_{f}(\sigma, \chi)$ has at most a simple zero in

$$
\sigma \geqq \frac{k+1}{2}-\frac{a}{\log q} \text {. }
$$

Proof. Let $\beta, \beta^{\prime}$ be two zeros of $L_{f}(\sigma, \chi)$ with $\beta^{\prime} \geqq \beta$. Following the proof of Lemma 6 with $\sigma_{4}=(k+1) / 2+a_{9} / \log q$ instead of $\sigma_{3}, \gamma=0$, we obtain for $a_{9}$ sufficiently small

Hence

$$
\frac{2}{\sigma_{4}-\beta}<\frac{3}{2} \frac{\log q}{a_{9}}
$$

$$
\beta<\frac{k+1}{2}-\frac{a}{\log q} \text {. }
$$

LEMMA 8. We have

$$
L_{f}\left(\frac{k+1}{2}, \chi\right) \neq 0 \quad \text { for all } \quad \chi \bmod q
$$

Proof. We use an idea due to Ogg [4]. In view of Lemma 5 and (2.17) we have to prove (2.22) only for $\chi^{2}=\chi_{0}, \chi \neq \chi_{0}$. Let

$$
h(s)=\zeta(s) L_{f}^{2}\left(s+\frac{k-1}{2}, \chi\right) \psi_{f}\left(s+k-1, \chi_{0}\right) L^{2}\left(s, \chi_{0}\right)
$$

Now $\psi_{f}\left(s+k-1, \chi_{0}\right) \zeta(s) L\left(s, \chi_{0}\right)$ is a holomorphic function except at $s=1$ (cf. [9]). If we suppose $L_{f}((k+1) / 2, \chi)=0$, then $h(s)$ is holomorphic in the whole plane. For $\sigma>1$ we have

$$
\log h(s)=\sum_{p, \nu \succeq 1} \frac{\left\{1+\alpha^{\nu}(p)+\overline{\alpha(p)}\right)^{\nu}}{\nu p^{\nu s}}=\sum_{n=2}^{\infty} \frac{c(n)}{n^{s}} \quad(\sigma>1),
$$

where $\alpha(p)=\chi(p) e^{i \theta_{p}(a)}$ and $c(n)$ is non-negative. If $\log h(s)$ were holomorphic in $\left[\sigma_{0}, 1\right)$, $\sigma_{0}<1$, then

$$
\log h(s)=\sum_{n=2}^{\infty} \frac{c(n)}{n^{s}} \text { for } \sigma>\sigma_{0}
$$


Let $\sigma_{0}$ be the largest real zero of $h(s)$. If it exists, $-\infty<\sigma_{0} \leqq 1$. In particular we have $\log |h(\sigma)|=\log h(\sigma) \geqq 0$ for $\sigma>\sigma_{0}$, which contradicts $h\left(\sigma_{0}\right)=0$. Hence $h(\sigma) \neq 0$. The functional equation (1.2) gives $L_{f}(0, \chi)=0$. Hence $s=-(k-1) / 2$ is a zero of $h(s)$, which contradicts $h(\sigma) \neq 0$. This proves $L_{f}((k+1) / 2, \chi) \neq 0$.

LEMMA 9. Let $\chi_{1}^{*}$ and $\chi_{2}^{*}$ be two real primitive characters $\bmod q_{1}^{*}$ and $q_{2}^{*}$ respectively with $\chi_{1}^{*} \neq \chi_{2}^{*}$. Then there is a $b_{1}$ such that at most one of the functions $L_{f}\left(s, \chi_{1}^{*}\right), L_{f}\left(s, \chi_{2}^{*}\right)$ has a simple zero for

$$
\sigma \geqq \frac{k+1}{2}-\frac{a}{\log \left(q_{1}^{*} q_{2}^{*}\right)}, \quad t=0 .
$$

Proof. First we may assume $\chi_{1}^{*}$ and $\chi_{2}^{*}$ to be different from $\chi_{0} \bmod 1$. Let $\chi_{1}$ and $\chi_{2}$ be the characters $\bmod q_{1}^{*} q_{2}^{*}$ induced by $\chi_{1}^{*} \bmod q_{1}^{*}$ and $\chi_{2}^{*} \bmod q_{2}^{*}$, respectively. Then $\chi_{1} \neq \chi_{0}, \chi_{2} \neq \chi_{0}, \chi_{1} \neq \chi_{2}, \chi_{1} \chi_{2} \neq \chi_{0}$. We prove Lemma 9 with $L_{f}\left(s, \chi_{1}\right), L_{f}\left(s, \chi_{2}\right)$ instead of $L_{f}\left(s, \chi_{1}^{*}\right), L_{f}\left(s, \chi_{2}^{*}\right)$, which is sufficient by Lemma 2 . We have

$$
\begin{aligned}
& -2 \frac{L^{\prime}}{L}\left(\sigma-\frac{k-1}{2}, \chi_{0}\right)-\frac{\psi_{f}^{\prime}}{\psi_{f}}\left(\sigma+\frac{k-1}{2}\right)-2 \frac{\zeta^{\prime}}{\zeta}\left(\sigma-\frac{k-1}{2}\right)-2 \frac{L_{f}^{\prime}}{L_{f}}\left(\sigma, \chi_{1}\right)-2 \frac{L_{f}^{\prime}}{L_{f}}\left(\sigma, \chi_{2}\right) \\
& -2 \frac{L^{\prime}}{L}\left(\sigma-\frac{k-1}{2}, \chi_{1} \chi_{2}\right)=\sum_{m \geqq 1, p} \frac{\log p}{p^{m(\sigma-(k-1) / 2)}}\left\{2 \chi_{0}\left(p^{m}\right)+4 \cos ^{2}\left(m \theta_{p}(a)\right)+4\left[\chi_{1}\left(p^{m}\right)\right.\right. \\
& \left.\left.+\chi_{2}\left(p^{m}\right)\right] \cos \left(m \theta_{p}(a)\right)+2 \chi_{1} \chi_{2}\left(p^{m}\right)\right\} \geqq 0 \quad\left(\sigma>\frac{k+1}{2}\right),
\end{aligned}
$$

since the expression in brackets is non-negative. Let

$$
\rho_{1}=\beta_{1}=\frac{k+1}{2}-\frac{a_{10}}{\log q}, \quad \beta_{2}=\beta_{2}=\frac{k+1}{2}+\frac{a_{11}}{\log q}
$$

be zeros of $L_{f}\left(s, X_{1}\right), L_{f}\left(s, X_{2}\right)$, respectively and

Then

$$
\sigma_{5}=\frac{k+1}{2}+\frac{a_{12}}{\log q} \text {. }
$$

$$
\begin{aligned}
& \frac{L_{f}^{\prime}}{L_{f}}\left(\alpha, \chi_{1}\right)>-A\left(\log q+\log \frac{1}{a_{12}}\right)+\frac{1}{\sigma_{5}-\beta_{1}} \\
& \frac{L_{f}^{\prime}}{L_{f}}\left(\sigma, \chi_{2}\right)>-A\left(\log q+\log \frac{1}{a_{12}}\right)+\frac{1}{\sigma_{5}-\beta_{2}} .
\end{aligned}
$$

We now take $\sigma_{5}$ instead of $\sigma_{0}$ in (2.12) and (2.15) and use the fact that

$$
-\frac{L^{\prime}}{L}\left(\sigma_{5}-\frac{k-1}{2}, \chi_{1} \chi_{2}\right)<A\left(\log q+\log \frac{1}{a_{12}}\right) .
$$


This together with (2.23) and (2.24) gives for sufficiently small $a_{12}$

$$
0 \leqq\left\{\frac{15}{4 a_{12}}-\frac{4}{a_{12}+\max \left(a_{10}, a_{11}\right)}\right\} \log q+A\left(\log q+\log \frac{1}{a_{12}}\right) \text {. }
$$

But for $a_{10}, a_{11}, a_{12}$ sufficiently small (2.25) cannot hold. Hence Lemma 9 follows.

LEMMA 10. Let $\chi_{1}^{*}$ and $\chi_{3}^{*}$ be two real primitive characters $\bmod q_{1}^{*}$ and $q_{3}^{*}$, respectively, where $q_{1}^{*} q_{3}^{*}>1$. Then there is a $b_{2}$, such that at most one of the functions $L_{f}\left(s, \chi_{1}^{*}\right)$, $L\left(s-(k-1) / 2, X_{3}^{*}\right)$ has a zero in

$$
\sigma \geqq \frac{k+1}{2}-\frac{b_{2}}{\log \left(q_{1}^{*} q_{3}^{*}\right)}, \quad t=0 .
$$

Proof. We may assume $\chi_{1}^{*} \neq \chi_{0}^{*}, \chi_{3} \neq \chi_{0}^{*}(\bmod 1)$. Let $\chi_{1}$ and $\chi_{3}$ be the characters $\bmod q=q_{1}^{*} q_{3}^{*}$ induced by $\chi_{1}^{*} \bmod q_{1}^{*}$ and $\chi_{3}^{*} \bmod q_{3}^{*}$ respectively. We prove Lemma 10 with $L_{f}\left(s, \chi_{1}\right), L\left(s-(k-1) / 2, \chi_{3}\right)$ instead of $L_{f}\left(s, \chi_{1}^{*}\right), L\left(s-(k-1) / 2, \chi_{3}^{*}\right)$ which is sufficient by Lemma 2 and the analogue for $L$-series. Let

and

$$
\begin{aligned}
& \rho_{3}=\beta_{3}=\frac{k+1}{2}+\frac{a_{13}}{\log q} \text { be a zero of } L_{f}\left(s, \chi_{1}\right), \\
& \rho_{4}=\beta_{4}=\frac{k+1}{2}+\frac{a_{14}}{\log q} \text { be a zero of } L\left(s-\frac{k-1}{2}, \chi_{3}\right)
\end{aligned}
$$

Then

$$
\sigma_{6}=\frac{k+1}{2}+\frac{a_{15}}{\log q} \text {. }
$$

$$
-\frac{L_{f}^{\prime}}{L_{f}}\left(\sigma_{6}, \chi_{1} \chi_{3}\right)<A\left(\log q+\log \frac{1}{a_{15}}\right) \text { for all } \chi_{1} \chi_{3} .
$$

Taking $\sigma=\sigma_{6}, \chi_{2}=\chi_{1} \chi_{3}$ in (2.23) we obtain as in the proof of Lemma 9 for sufficiently small $a_{15}$

$$
0 \leqq\left\{\frac{15}{4 a_{15}}-\frac{4}{a_{15}+\max \left(a_{13}, a_{14}\right)}\right\} \log q+A\left(\log q+\log \frac{1}{a_{15}}\right) .
$$

But for $a_{13}, a_{14}, a_{15}$ small enough, (2.26) cannot hold. Hence Lemma 10 follows. We now turn to the proofs of Theorem 1 and Theorem 2 .

Proof of Theorem 1. In view of the above lemmas it is sufficient to prove the following: for $z \geqq 2$ there is a $c_{1}$ independent of $z$, such that $L_{f}(s, \chi) \neq 0$ for $\sigma \geqq$ $(k+1) / 2-c_{1} / \log z, t=0$, for all real primitive $\chi^{*} \bmod q^{*}$ where $q^{*} \leqq z$ with at most one exception. If $\chi_{1}^{*} \bmod q_{1}^{*}$ and $\chi_{2}^{*} \bmod q_{2}^{*}$ were two such characters, then Lemma 9 shows that at most one of the functions $L_{f}\left(s, \chi_{1}^{*}\right), L_{f}\left(s, \chi_{2}^{*}\right)$ has a zero for

$$
\sigma \geqq \frac{k+1}{2}-\frac{b_{1}}{2 \log z} \geqq \frac{k+1}{2}-\frac{b_{1}}{\log \left(q_{1}^{*} q_{2}^{*}\right)}, \quad t=0 \text {. }
$$


Proof of Theorem 2. In view of Theorem 1 and [5], IV, Satz 6.9, it is sufficient, to prove the following: for $z \geqq 2$ there is a $c_{2}$ independent of $z$, such that at most one of the functions $L_{f}\left(s, \chi^{*}\right)$ and $L\left(s-(k-1) / 2, \hat{\chi}^{*}\right)$ with real primitive characters $\chi^{*} \bmod q^{*}$, $\hat{\chi}^{*} \bmod \hat{q}^{*}$ with $q^{*} \leqq z, \hat{q}^{*} \leqq z$ has a zero in $\sigma \geqq(k+1) / 2-a / \log z, t=0$. But this follows from Lemma 10.

\section{Proofs of Theorem 3 and Theorem 4.}

Proof of Theorem 3. We may assume that the Siegel zero exists, otherwise part (i) of Theorem 3 holds (cf. [5]). Let

$$
\begin{aligned}
& \Lambda_{a}(n)= \begin{cases}2 \cos \left(m \theta_{p}(a)\right) \log p & \left(n=p^{m}, m \in \mathbb{N}\right) \\
0 & \text { (otherwise) }\end{cases} \\
& \psi_{a}(x, \chi)=\sum_{n \leq x} \chi(n) \Lambda_{a}(n), \\
& \psi_{a}(x, q, l)=\sum_{\substack{n \leqq x \\
n=l(q)}} \Lambda_{a}(n) \text { for } \quad(q, l)=1
\end{aligned}
$$

Then

$$
\psi_{a}(x, q, l)=\frac{1}{\varphi(q)} \sum_{x \bmod q} \bar{\chi}(l) \psi_{a}(x, \chi)
$$

and by Perron's formula

where

$$
\sum_{n \leq x} \chi(n) \Lambda_{a}(n)=-\frac{1}{2 \pi i} \int_{c-i T}^{c+i T} \frac{L_{f}^{\prime}}{L_{f}}(s, \chi) \frac{x^{s}-(k-1) / 2}{s-(k-1) / 2} d s+O\left(\frac{x}{T} \log ^{2} x\right)
$$

$$
c=\frac{1}{2}(k+1)+\frac{1}{\log x} \text { for } T \leqq 2 \text { and } x=[x]+\frac{1}{2} .
$$

Let $\Gamma_{1}, \Gamma_{2}, \Gamma_{3}$ be the following curves in the complex plane.

$$
\begin{aligned}
& \Gamma_{1}: s=\frac{k+1}{2}+\sigma+i T, \quad-\frac{b_{3}}{3 \log (q(T+2))} \leqq \sigma \leqq \frac{1}{\log x} . \\
& \Gamma_{2}: s=\frac{k+1}{2}-\frac{b_{3}}{3 \log (q(|t|+2))}-i t, \quad-T \leqq t \leqq T . \\
& \Gamma_{3}: s=\frac{k+1}{2}+\sigma-i T, \quad-\frac{b_{3}}{3 \log (q(T+2))} \leqq \sigma \leqq \frac{1}{\log x} .
\end{aligned}
$$

Applying Satz 4.6 (Anhang) of [5] with

$$
r=\frac{1}{2}, \quad r_{1}=\frac{2 b_{3}}{3 \log (q(|t|+2))}, \quad s_{0}=\frac{k+1}{2}-\frac{b_{3}}{3 \log (q(|t|+2)}+i t
$$


for sufficiently small $b_{3}$, we obtain

for

$$
\frac{L_{f}^{\prime}}{L_{f}}(s, \chi)=O(\log (q(|t|+2)))
$$

For

$$
\frac{k+1}{2}-\frac{b_{3}}{3 \log (q(|t|+2))} \leqq \sigma \leqq \frac{k+1}{2}+\frac{b_{3}}{\log (q(|t|+2))}, \quad t \in \mathbb{R} .
$$

(3.3) follows since

$$
\sigma>\frac{k+1}{2}+\frac{b_{3}}{\log (q(|t|+2))}
$$

$$
\left|\frac{L_{f}^{\prime}}{L_{f}}(s, x)\right| \leqq \sum_{n=1}^{\infty} \frac{\Lambda_{a}(n)}{n^{\sigma-(k-1) / 2}} \leqq-2 \frac{\zeta^{\prime}}{\zeta}\left(\sigma-\frac{k-1}{2}\right) \leqq \frac{4}{\sigma-\frac{k+1}{2}} \quad\left(\sigma>\frac{k+1}{2}\right)
$$

(3.2) and Cauchy's theorem now gives

$$
\sum_{n \leq x} \chi(n) \Lambda_{a}(n)=O\left(\left|\int_{\Gamma_{1}-\Gamma_{2}-\Gamma_{3}} \frac{L_{f}^{\prime}}{L_{f}}(s, \chi) \frac{x^{s-(k-1) / 2}}{s-(k-1) / 2} d s\right|\right)+O\left(\frac{x}{T} \log ^{2} x\right)
$$

Then, by (3.3),

$$
\begin{gathered}
\int_{\Gamma_{1}-\Gamma_{3}} \frac{L_{f}^{\prime}}{L_{f}}(s, \chi) \frac{x^{s-(k-1) / 2}}{s-\frac{k-1}{2}} d s=O\left\{\frac{x}{T} \log (q T)\right\}, \\
\int_{\Gamma_{2}} \frac{L_{f}^{\prime}}{L_{f}}(s, \chi) \frac{x^{s-(k-1) / 2}}{s-\frac{k-1}{2}} d s=O\left(\exp \left\{\left(1-\frac{b_{3}}{3 \log (q(T+2))}\right) \log x\right\} \cdot \log ^{2}(q T)\right)
\end{gathered}
$$

Here now take $\log T=\sqrt{ } \log x,(3.4)$ and the inequality $\log q \leqq B \sqrt{ } \log x$ give

$$
\sum_{n \leqq x} \chi(n) \Lambda_{a}(n)=O\left(x \exp \left\{-c_{3} \sqrt{ } \log x\right\}\right)
$$

Hence, by (3.1),

$$
\psi_{a}(x, q, l)=O\left(x \exp \left\{-c_{4} \sqrt{ } \log x\right\}, \text { for } q \leqq \exp \{B \sqrt{ } \log x\} \text { and } x \geqq 2 .\right.
$$

We note that

$$
\sum_{\substack{p \leq x \\ p \operatorname{mal}(q)}} a(p) p^{-(k-1) / 2} \log p=\psi_{a}(x, q, l)+O\left(\sum_{\substack{\nu \\ p \leq x \\ \nu \geqq 2}} \log p\right)=O\left(x \exp \left\{-c_{4} \sqrt{ } \log x\right\}\right) .
$$

This completes the proofs. 
Proof of Theorem 4. The proof proceeds as in the proof of Theorem 3. The condition

$$
L_{a}(s, \chi) \neq 0 \text { for } \quad \sigma \geqq \frac{k+1}{2}-\frac{a}{\log (q(|t|+2))}
$$

(which in the proof of Theorem 3 follows from Theorem 2) holds now for fixed $q$ by Lemmas 1, 4, 5, 6 and 8 .

\section{REFERENCES}

1. P. Deligne, La Conjecture de Weil I. Inst. Hautes Etudes Sci. Publ. Math. 43 (1973), 273-307.

2. C. G. Lekkerkerker, On the zeros of a class of Dirichlet series (Van Gorcum \& Comp., Assen, 1955).

3. C. J. Moreno, Prime number theorems for the coefficients of modular forms, Bull. Amer. Math. Soc. 78 (1972), 796-798.

4. A. P. Ogg, On a convolution of $L$-series Invent. Math. 7 (1969), 297-312.

5. K. Pracher, Primzahlverteilung (Springer, 1957).

6. H. Rademacher, On the Phragmen-Lindelöf theorem and some applications. Math. Z. 72 (1959), 192-204.

7. R. A. Rankin, Contributions to the theory of Ramanujan's function $\tau(n)$ and similar arithmetical functions. I. The zeros of the function $\sum_{n=1}^{\infty} \tau(n) / n^{s}$ on the line $\operatorname{Re} s=\frac{13}{2}$, Proc. Cambridge
Phil. Soc. 35 (1939), 351-356.

8. R. A. Rankin, Contributions to the theory of Ramanujan's function $\tau(n)$ and similar arithmetical functions. II The order of the Fourier coefficients of integral modular forms, Proc. Cambridge Phil. Soc. 35 (1939), 357-372.

9. R. A. Rankin, An $\Omega$-Result for the coefficients of cusp forms. Math. Ann. 203 (1973), 239-250.

UNIVERSITÄT ULM

ABTEILUNG FÜr MATHEMATIK

OBERER ESELSBERG

D-7900 ULM 